\title{
Effect of HK2, PKM2 and LDHA on Cetuximab efficacy in metastatic colorectal cancer
}

\author{
HAOHUA WANG ${ }^{1-3^{*}}$, ROUJUN PENG ${ }^{1-3 *}$, XIUXING CHEN ${ }^{1-3}$, RUI JIA ${ }^{2-4}$, CHUNYUE HUANG $^{2-4}$, \\ YUANYUAN HUANG ${ }^{1-3}$, LIANGPING XIA ${ }^{1-3}$ and GUIFANG GUO ${ }^{1-3}$ \\ ${ }^{1}$ VIP Region; ${ }^{2}$ State Key Laboratory of Oncology in South China; ${ }^{3}$ Collaborative Innovation Center for Cancer Medicine; \\ ${ }^{4}$ Radiation Region, Sun Yat-sen University Cancer Center, Guangzhou, Guangdong 510060, P.R. China
}

Received August 12, 2017; Accepted January 11, 2018

DOI: $10.3892 / 01.2018 .8005$

\begin{abstract}
Although hexokinase (HK) 2, pyruvate kinase muscle (PKM) isozyme 2 and lactate dehydrogenase (LDH) A predict the efficacy of medicines in various solid tumors, their ability to predict the efficacy of cetuximab in metastatic colorectal cancer (mCRC) remains unclear. mCRC patients with pathological specimens who received cetuximab and chemotherapy from 2005 to 2015 in the present institution were enrolled. Immunohistochemistry was used to detect HK2, PKM2 and LDHA expression. SPSS20 was used for statistical analysis. A total of 68 patients were included; 33 received cetuximab plus chemotherapy as first-line therapy, and the rest, as second- or later-line therapy. HK2 expression levels were increased in cancer compared with normal tissue (75.4\% vs. 40\%; $\mathrm{P}<0.001)$, however PKM2 ( $\mathrm{P}=0.243)$ and LDHA $(\mathrm{P}=0.067)$ expression levels were not. For progression-free survival (PFS) with first-line cetuximab plus chemotherapy, patients with high HK2 expression exhibited longer PFS compared with those with low HK2 expression (23.9 months vs. 6.9 months; $\mathrm{P}=0.021)$. However, this positive association was absent in 35 cases administered first-line chemotherapy alone (13.4 months vs. 13.5 months; $\mathrm{P}=0.539$ ). LDHA expression was associated with the PFS of patients receiving first-line chemotherapy (18.3 and 10.1 months for high and low expression, respectively; $\mathrm{P}=0.005)$, whereas this association was absent in cetuximab plus chemotherapy cases (19.9 months vs. 12 months; $\mathrm{P}=0.522)$. Furthermore, high LDHA expression correlated with high overall response rate $(\mathrm{ORR})(72.2 \%$ vs. $15.4 \%, \mathrm{P}=0.006)$ for chemotherapy, however not disease control rate (DCR) $(\mathrm{P}=0.074)$. Neither
\end{abstract}

Correspondence to: Dr Guifang Guo or Dr Liangping Xia, VIP Region, Sun Yat-sen University Cancer Center, 651 Dongfeng Road East, Guangzhou, Guangdong 510060, P.R. China

E-mail: gguifang@163.com

E-mail: xialp@sysucc.org.cn

*Contributed equally

Key words: cetuximab, HK-II, PKM2, LDHA, mCRC
DCR nor ORR were associated with HK2 expression. PKM2 expression did not affect PFS, DCR or ORR. LDHA expression $(\mathrm{P}=0.005)$, pathological differentiation $(\mathrm{P}=0.019)$ and synchronous/metachronous metastasis $(\mathrm{P}=0.014)$ were independent predictive factors of PFS for all first-line patients, and tumor differentiation $(\mathrm{P}=0.002)$ was associated with overall survival (OS) in multivariate analysis. HK2, PKM2 and LDHA did not impact OS. It was concluded that HK2 expression was increased in colorectal cancer tissue and may predict cetuximab efficacy and LDHA for chemotherapy treatment of $\mathrm{mCRC}$.

\section{Introduction}

Colorectal cancer (CRC) is the third most commonly diagnosed cancer in men and the second most common in women worldwide, with an estimated 1.4 million cases and 693,900 deaths occurring in 2012 (1). In several Asian countries, the incidence of $\mathrm{CRC}$ is increasing, which may be related to an increased prevalence of risk factors for $\mathrm{CRC}$, including unhealthy diet, obesity, and smoking $(2,3)$. Although early CRC diagnosis and treatment strategies have improved in recent decades, most patients still present with advanced disease at diagnosis, and their prognoses remain poor (4). The main reason is that $\mathrm{CRC}$ is usually diagnosed at late stages, often with lymph node or distant metastases. In the past decade, the survival of metastatic CRC patients has significantly improved due to the development of new combinations of chemotherapy drugs, including 5-fluorouracil, irinotecan, and oxaliplatin. In particular, the introduction of new targeted therapies such as monoclonal antibodies (cetuximab and panitumumab) against epidermal growth factor receptor (EGFR) or monoclonal antibodies (bevacizumab, ramucirumab, and aflibercept) against vascular endothelial growth factor has made a large impact. The addition of targeted therapies to chemotherapy regimens results in increased toxicity and treatment costs (5) and therefore requires the identification of markers to identify patients who are likely to respond to them. The chimeric IgG1 monoclonal antibody cetuximab is effective for patients with wild-type RAS $(6,7)$ and left-side CRC patients $(8,9)$. However, the response rate $(\mathrm{RR})$ is $40-60 \%$ for CRC patients with wild-type RAS. Therefore, approximately $50 \%$ of patients with wild-type RAS are still resistant 
to cetuximab. Identifying a novel predictor for improving therapy would be significant. In the present study, we explored new predictive markers correlated with cetuximab efficacy in metastatic CRC (mCRC).

SGLT1, an active glucose transporter that maintains enough glucose for cell survival, was downregulated by EGFR knockdown in cancer cells (10). Cetuximab, which targets EGFR, may also affect SGLT1 function and glucose metabolism. Our previous study found that SGLT1 expression $(\mathrm{P}<0.010)$ was higher in colon cancer tissues than in normal tissues and was related to clinical stage $(\mathrm{P}=0.030)(11)$. We found that in an in vitro colon cancer cell line, cetuximab treatment downregulated hexokinase 2 (HK2), the key enzyme for aerobic glycolysis. Based on these results, we considered it significant to study the connection between aerobic glycolysis and cetuximab efficacy in colon cancer.

Aerobic glycolysis is a hallmark of cancer cells, which exhibit lactate production even in the presence of ample oxygen, a phenomenon known as the Warburg effect (12). An important advantage of the increased glycolysis of tumor cells is the production of energy without the consumption of oxygen and glycolytic intermediates, such as amino acids, nucleotides, phospholipids and triglycerides, which are used as macromolecules to synthesize structural elements for new cells. Three key enzymes, namely, HK2, pyruvate kinase muscle isozyme M2 (PKM2) and lactate dehydrogenase A (LDHA), are critical glycolysis regulators. Although the expression of HK2, PKM2 and LDHA has been individually reported to be correlated with cancer cell growth, their predictive role in patients with mCRC remains unclear. In the present study, we evaluated the clinical value of these glycolytic enzymes in predicting the effect of cetuximab on mCRC. HK2, PKM2 and LDHA expression in CRC and normal tissue was compared. The correlation of their expression with clinical pathological features, the progression-free survival (PFS) of first-line palliative therapy and survival was evaluated to explore their predictive and prognostic values for CRC treated with cetuximab.

\section{Patients and methods}

Patient eligibility. Patients with $\mathrm{mCRC}$ who had ever been administered cetuximab combined with first-line or later chemotherapy at our institution between January 1, 2005, and October 1, 2015, were identified. Although Cetuximab was first introduced into China in 2006, some people had enough money to buy cetuximab in 2005 in Hong Kong. That is why patients in the present study accepted cetuximab during 2005-2015. At first, 235 patients were enrolled, but only 68 patients with paraffin embedding tissue were available for immunohistochemical testing. All of the eligible patients in the present study satisfied the following requirements: i) intact medical data, ii) explicit pathological diagnosis of colon cancer, iii) at least two experts confirming metastasis based on clinical and imaging methods, iv) at least two cycles of palliative chemotherapy with or without first-line cetuximab and v) sufficient cancer specimens in the archives of the Tissue Bank to test HK2, PKM2 and LDHA expression. Furthermore, we collected negative margin specimens from most patients as normal tissue. Written informed consent was received from the sample donors, and approval was granted by the Institute Research Medical Ethics Committee of Sun Yat-sen University (Guangzhou, China). The basic clinical characteristics for all of the patients are reported in Table I.

Immunohistochemistry (IHC). Standard immunohistochemical procedures were performed to determine the location and levels of HK2, PKM2 and LDHA expression in CRCs. Briefly, formalin-fixed paraffin-embedded (FFPE) tissue blocks were cut into $4 \mu \mathrm{m}$ sections for IHC and hematoxylin and eosin staining. Polyclonal rabbit anti-HK2 antibody, polyclonal rabbit anti-PKM2 antibody or polyclonal rabbit anti-LDHA antibody (all Cell Signaling Technology, Danvers, MA, USA) diluted 1:150 was used in the present study. Tissue sections were de-waxed in xylene, rehydrated through a graded ethanol series, and incubated in retrieval buffer solution for antigen recovery. Next, the samples were incubated with hydrogen peroxide for $10 \mathrm{~min}$ to block intrinsic peroxidases, washed in phosphate buffered saline (PBS), and blocked with normal serum for $10 \mathrm{~min}$. The samples were incubated with a primary antibody for $40 \mathrm{~min}$ at $37^{\circ} \mathrm{C}$ followed by EnVision тм Detection systems (DAKO; Agilent Technologies, Inc., Glostrup, Denmark) for $30 \mathrm{~min}$ at $37^{\circ} \mathrm{C}$. The signal was visualized with diaminobenzidine (DAB). Negative controls were obtained by substituting non-immune rabbit serum for the primary antibodies.

Evaluation of immunostaining results. Protein expression was evaluated by two individuals using an Olympus CX31 microscope (Olympus, Center Valley, PA). The mean percentage of positive tumor cells was determined from five areas at magnification, $\mathrm{x} 400$ and assigned a $0-100 \%$ value. The intensity of immunostaining was scored as follows: negative, 0; weak, 1; moderate, 2; and intense, 3. For the percentage of positive tumor cells, the score was classified as $0-4(10 \%, 11-25 \%, 26-50 \%, 51-75 \%,>75 \%)$. Thus, the percentage of positive tumor cells and the staining intensity were multiplied to produce a total score for each sample. Then, the total HK2 and PKM2 scores were categorized as low expression ( $\leq 3)$ and high expression (>3) (13). The total scores for LDHA were categorized as low expression $(<6)$ and high expression $(\geq 6)$.

Data analysis. IBM SPSS 20 software was used to perform statistical analyses. Associations between discrete variables were assessed using the chi-square test or Fisher's exact test as appropriate. The statistical test used to analyze data derived from such small cohorts is Chi square test for continuity correction (14). The Kaplan-Meier method was used to estimate tumor progression in $\mathrm{mCRC}$, and the log-rank test was used to determine the statistical significance. The groups were compared with respect to survival using Cox regression analysis. Hazard ratios were determined using univariate and multivariate Cox regression analysis. $\mathrm{P}<0.05$ was considered to indicate a statistically significant difference.

\section{Results}

Basic patient characteristics. A total of 68 patients consisting of 42 men $(61.8 \%)$ and 26 women $(38.2 \%)$ were included 
Table I. Correlation between HK2, PKM2 and LDHA expression and clinicopathological characteristics in CRC patients.

\begin{tabular}{|c|c|c|c|c|c|c|c|c|c|c|}
\hline \multirow[b]{2}{*}{ Variable } & \multirow[b]{2}{*}{$\mathrm{N}=68(\%)$} & \multicolumn{3}{|c|}{ HK2 expression } & \multicolumn{3}{|c|}{ PKM2 expression } & \multicolumn{3}{|c|}{ LDHA expression } \\
\hline & & Low & High & $\mathrm{P}$ & Low & High & $\mathrm{P}$ & Low & High & P-value \\
\hline Sex & & & & 0.663 & & & 0.973 & & & 0.891 \\
\hline Male & $44(64.7)$ & 10 & 28 & & 11 & 28 & & 15 & 21 & \\
\hline Female & $24(35.3)$ & 4 & 15 & & 5 & 13 & & 10 & 13 & \\
\hline Age, years & & & & 0.807 & & & 0.829 & & & 0.549 \\
\hline$<65$ & $55(80.9)$ & 11 & 37 & & 13 & 36 & & 19 & 28 & \\
\hline$\geq 65$ & $13(19.1)$ & 3 & 6 & & 3 & 5 & & 6 & 6 & \\
\hline Family history of cancer & & & & 0.816 & & & 0.236 & & & 0.819 \\
\hline No & $55(80.9)$ & 11 & 35 & & 15 & 31 & & 20 & 28 & \\
\hline Yes & $13(19.1)$ & 3 & 8 & & 1 & 10 & & 5 & 6 & \\
\hline Tumor location & & & & 0.895 & & & 0.685 & & & 0.935 \\
\hline Left-side & $41(60.3)$ & 7 & 28 & & 10 & 27 & & 18 & 19 & \\
\hline Right-side & $14(20.6)$ & 2 & 9 & & 4 & 6 & & 6 & 6 & \\
\hline NA & $13(19.1)$ & & & & & & & & & \\
\hline Pathological differentiation & & & & 0.298 & & & 0.955 & & & 0.623 \\
\hline Low & $17(25.0)$ & 5 & 8 & & 4 & 10 & & 6 & 10 & \\
\hline Median + high & $44(64.7)$ & 7 & 30 & & 10 & 26 & & 17 & 21 & \\
\hline NA & $7(10.3)$ & & & & & & & & & \\
\hline T stage & & & & 0.780 & & & 0.747 & & & 0.07 \\
\hline $\mathrm{T} 1+\mathrm{T} 2+\mathrm{T} 3$ & $41(60.3)$ & 9 & 26 & & 11 & 25 & & 11 & 23 & \\
\hline $\mathrm{T} 4$ & $22(32.4)$ & 4 & 14 & & 4 & 14 & & 12 & 9 & \\
\hline NA & $5(7.4)$ & & & & & & & & & \\
\hline $\mathrm{N}$ stage & & & & 0.15 & & & 0.839 & & & 0.903 \\
\hline N0 & $22(32.4)$ & 3 & 18 & & 4 & 16 & & 7 & 10 & \\
\hline $\mathrm{N} 1+\mathrm{N} 2$ & $36(52.9)$ & 9 & 19 & & 8 & 22 & & 13 & 20 & \\
\hline NA & $10(14.7)$ & & & & & & & & & \\
\hline Synchronous/metachronous metastasis & & & & 0.091 & & & 0.586 & & & 0.569 \\
\hline $\mathrm{I}+\mathrm{II}+\mathrm{III}$ & $23(33.8)$ & 8 & 13 & & 7 & 14 & & 7 & 12 & \\
\hline IV & $43(63.2)$ & 6 & 28 & & 9 & 25 & & 17 & 21 & \\
\hline NA & $2(2.9)$ & & & & & & & & & \\
\hline
\end{tabular}

HK2, hexokinase 2; PKM2, pyruvate kinase muscle isozyme M2; LDHA, lactate dehydrogenase A; CRC, colorectal cancer; NA, not applicable.

in the present study. The age of the patients ranged from 23 to 77 years, with a median age of 54.5 years. Overall survival (OS) was analyzed in 68 patients $(98.5 \%)$, whereas PFS was assessed in 63 patients (92.6\%). The median PFS for first-line chemotherapy was 7.4 months (0.5-32.9), and the median OS was 29.9 months (2.2-137). The objective response rate (ORR) and disease control rate (DCR) were 47.8 and $71.6 \%$ based on data from 67 patients. The basic clinical and pathological characteristics of all the cases and their correlations with HK2, PKM2 and LDHA are shown in Table I. Both $\mathrm{N} 1$ and N2 indicated lymph node metastasis. Besides, the total cases was small, separating N1 and N2 will make the subgroup number smaller. Therefore we combined N1 and N2 as a group.

$H K 2$ is significantly upregulated in CRC tissues. According to the quantitative scoring method described before, HK2,
PKM2 and LDHA were divided into high expression and low expression. As shown in Fig. 1, HK2 expression was significantly upregulated in tumor specimens compared to normal specimens $(75.4 \%$ vs. $40 \%, \mathrm{P}<0.001)$. However, PKM2 and LDHA expression did not differ between CRC and normal tissue $(71.9 \%$ vs. $61.2 \%, \mathrm{P}=0.243$ and $57.6 \%$ vs. $40 \%$, $\mathrm{P}=0.067)$. The overall average in expression has no difference, but there is still some people have strong staining in cancer tissue and low staining in normal tissue. We performed immunohistochemical staining for HK2, LDHA and PKM2 in CRC samples and in negative margins as a normal tissue control (Fig. 2).

Correlation between HK2, PKM2 and LDHA expression and clinicopathological parameters in CRC patients. The correlation between $\mathrm{HK} 2$ expression and the corresponding clinicopathological parameters of CRC patients were 


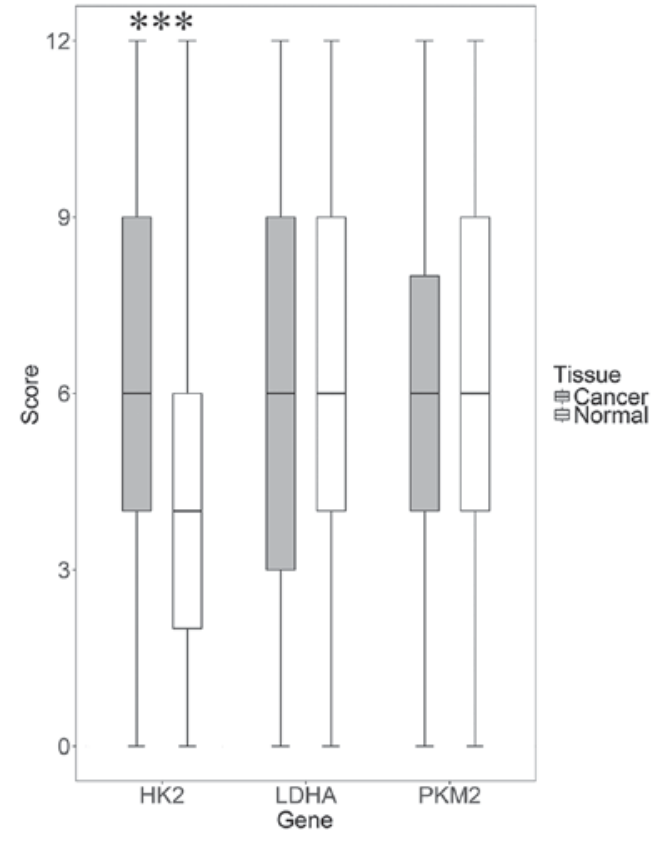

Figure 1. The different expression levels of HK2 ( $\mathrm{P}<0.001)$, LDHA $(\mathrm{P}=0.067)$ and PKM2 $(\mathrm{P}=0.243)$ tested by immunohistochemical staining in CRC samples compared to normal tissue. ${ }^{* * * *} \mathrm{P}<0.001$. HK2, hexokinase 2; PKM2, pyruvate kinase muscle isozyme M2; LDHA, lactate dehydrogenase A; CRC, colorectal cancer.

calculated using the Pearson chi-square test. The results are shown in Table I. HK2, PKM2 and LDHA expression was not significantly correlated with sex, age, family history of cancer, tumor location, pathological differentiation, $\mathrm{T}$ stage, $\mathrm{N}$ stage or synchronous/metachronous metastasis.

Predictive value of HK2, PKM2 and LDHA expression in CRC patients treated with cetuximab. We studied the correlation of HK2, PKM2 and LDHA in cancer tissue with PFS in two groups: one group of 33 patients treated with cetuximab plus first-line chemotherapy and another group of 35 cases administered chemotherapy alone. As shown in Table II, when a combination of cetuximab and chemotherapy was administered, patients with high HK2 expression had longer first-line PFS than those patients with low HK2 expression (23.9 months vs. 6.9 months; $\mathrm{P}=0.021$ ) (Fig. 3A). However, the PFS of patients receiving chemotherapy alone did not differ between the groups with high HK2 expression and low HK2 expression (13.4 months vs. 13.5 months, $\mathrm{P}=0.539$ ) (Fig. 3B). In contrast to HK2, LDHA expression was associated with first-line PFS in patients treated with chemotherapy alone, with a PFS of 18.3 and 10.1 months for high and low expression, respectively $(\mathrm{P}=0.005)$ (Fig. 3D), whereas this relationship was not observed in cases treated with first-line cetuximab plus chemotherapy (19.9 months vs. 12 months, $\mathrm{P}=0.522$ ) (Fig. 3C). Furthermore, high LDHA expression correlated with high ORR $(72.2 \%$ vs. $15.4 \%, \mathrm{P}=0.006)$ but not DCR ( $88.9 \%$ vs. $53.8 \%, \mathrm{P}=0.074)$ in patients who received chemotherapy. Neither DCR nor ORR was related to HK2 expression regardless of first-line therapy with or without cetuximab. Additionally, PKM2 expression had no effect on PFS, DCR or ORR for first-line therapy with or without cetuximab.
A

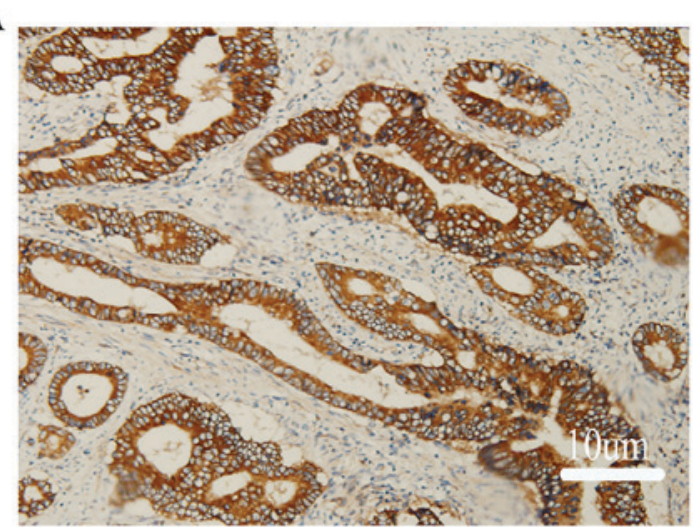

B

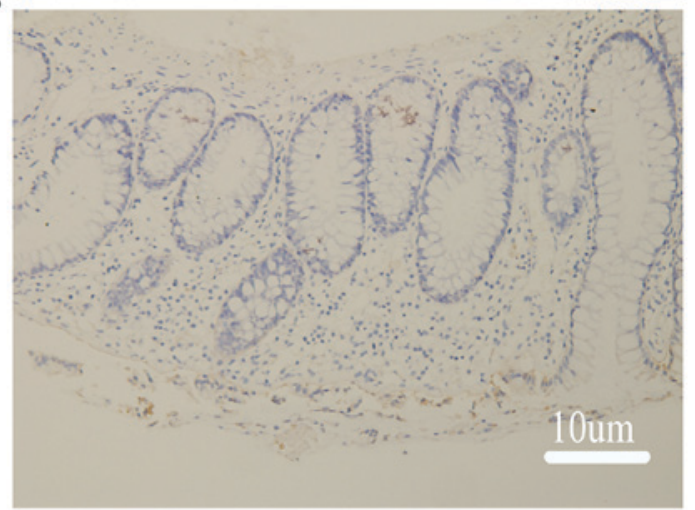

Figure 2. Immunohistochemical staining. (A) High HK2 expression in CRC tissue; (B) low HK2 expression in normal tissue. HK2, hexokinase 2; CRC, colorectal cancer.

Prognostic value of $H K 2, P K M 2$ and LDHA in $m C R C$ patients for cetuximab and chemotherapy treatment. For all patients, HK2, PKM2 and LDHA had no impact on OS $(\mathrm{P}=0.462, \mathrm{P}=0.418$ and $\mathrm{P}=0.243)$ by the Kaplan-Meier method. By univariate Cox regression analysis, LDHA expression $(\mathrm{P}=0.016)$ and tumor site $(\mathrm{P}=0.035)$ were risk factors for first-line PFS, and pathological differentiation $(\mathrm{P}<0.001)$ and synchronous/metachronous metastasis $(\mathrm{P}=0.043)$ were risk factors affecting OS. Multivariate Cox regression analysis revealed that LDHA expression $(\mathrm{P}=0.005)$, pathological differentiation $(\mathrm{P}=0.019)$ and synchronous/metachronous metastasis $(\mathrm{P}=0.014)$ were independent prognostic factors for PFS, and pathological differentiation was a prognostic factor for OS $(\mathrm{P}=0.002)$. The detailed data are shown in Table III.

\section{Discussion}

Although many new predictive markers have been found in other cancers in the era of precision medicine, targeted therapy for $\mathrm{mCRC}$ has shown little progress. Therefore, understanding how to assess known target molecules is important for treating mCRC. We evaluated the role of the key glycolytic enzymes HK2, PKM2 and LDHA for predicting cetuximab efficacy in $\mathrm{mCRC}$. We found that HK2 expression was higher in CRC tissue. HK2 predicts cetuximab efficacy as a first-line therapy, and LDHA is predictive for chemotherapy. PKM2 did not predict cetuximab efficacy. For patients who received cetuximab as first- or later-line treatment, LDHA expression, 
Table II. Relationship of HK2, PKM2 and LDHA with the short-efficacy of cetuximab plus chemotherapy vs. chemotherapy alone in the first line of $\mathrm{mCRC}$ patients.

\begin{tabular}{|c|c|c|c|c|c|c|c|c|}
\hline \multirow[b]{2}{*}{ Subgroup/factors } & \multicolumn{2}{|c|}{ Factors } & \multicolumn{2}{|c|}{ DCR } & \multicolumn{2}{|c|}{ ORR } & \multicolumn{2}{|c|}{ PFS } \\
\hline & Expression & $\mathrm{N}(\%)$ & $\mathrm{N}(\%)$ & $\mathrm{P}$ & $\mathrm{N}(\%)$ & $\mathrm{P}$ & Months & P-value \\
\hline Chemotherapy plus cetuximab & & 33 & & & & & & \\
\hline HK2 & $\begin{array}{l}\text { High } \\
\text { Low } \\
\text { NA }\end{array}$ & $\begin{array}{c}19(57.6) \\
9(27.3) \\
5(15.1)\end{array}$ & $\begin{array}{r}16(84.2) \\
5(55.6)\end{array}$ & 0.102 & $\begin{array}{r}10(52.6) \\
4(44.4)\end{array}$ & 0.686 & $\begin{array}{c}23.9 \\
6.9\end{array}$ & 0.021 \\
\hline PKM2 & $\begin{array}{l}\text { High } \\
\text { Low } \\
\text { NA }\end{array}$ & $\begin{array}{c}19(57.6) \\
7(21.2) \\
7(21.2)\end{array}$ & $\begin{array}{r}14(73.7) \\
4(57.1)\end{array}$ & 0.740 & $\begin{array}{r}10(52.6) \\
3(42.9)\end{array}$ & 0.658 & $\begin{array}{l}22.1 \\
12.8\end{array}$ & 0.84 \\
\hline LDHA & $\begin{array}{l}\text { High } \\
\text { Low } \\
\text { NA }\end{array}$ & $\begin{array}{c}16(48.5) \\
12(36.4) \\
5(15.1)\end{array}$ & $\begin{array}{r}10(62.5) \\
9(75.0)\end{array}$ & 0.962 & $\begin{array}{l}5(41.7) \\
6(40.0)\end{array}$ & 0.930 & $\begin{array}{c}19.9 \\
12\end{array}$ & 0.522 \\
\hline Chemotherapy alone & & 35 & & & & & & \\
\hline HK2 & $\begin{array}{l}\text { High } \\
\text { Low } \\
\text { NA }\end{array}$ & $\begin{array}{c}24(68.6) \\
5(14.3) \\
6(17.1)\end{array}$ & $\begin{array}{r}15(62.5) \\
4(80.0)\end{array}$ & 0.817 & $\begin{array}{r}10(41.7) \\
4(80.0)\end{array}$ & 0.285 & $\begin{array}{l}13.4 \\
13.5\end{array}$ & 0.539 \\
\hline PKM2 & $\begin{array}{l}\text { High } \\
\text { Low } \\
\text { NA }\end{array}$ & $\begin{array}{c}22(62.9) \\
9(25.7) \\
4(11.4)\end{array}$ & $\begin{array}{r}16(72.7) \\
5(55.6)\end{array}$ & 0.613 & $\begin{array}{r}11(50.0) \\
4(44.4)\end{array}$ & 0.779 & $\begin{array}{l}15.1 \\
12.8\end{array}$ & 0.331 \\
\hline LDHA & $\begin{array}{l}\text { High } \\
\text { Low } \\
\text { NA }\end{array}$ & $\begin{array}{c}18(51.5) \\
13(37.1) \\
4(11.4)\end{array}$ & $\begin{array}{r}16(88.9) \\
7(53.8)\end{array}$ & 0.074 & $\begin{array}{r}13(72.2) \\
2(15.4)\end{array}$ & 0.006 & $\begin{array}{l}18.3 \\
10.1\end{array}$ & 0.005 \\
\hline
\end{tabular}

Bold values indicate $\mathrm{P}<0.05$. HK2, hexokinase 2; PKM2, pyruvate kinase muscle isozyme M2; LDHA, lactate dehydrogenase A; CRC, colorectal cancer; DCR, disease control rate; ORR, overall response rate; PFS, progression-free survival; NA, not applicable.

pathological differentiation and synchronous/metachronous metastasis were independent prognostic factors for PFS, and pathological differentiation was an independent prognostic factor for OS. HK2, PKM2 and LDHA did not impact OS.

Some studies have reported that HK2 expression was higher in tumor tissue than adjacent normal tissue in many malignant tumor types, such as gastric cancers (15), renal cell carcinomas (16), and hepatocellular carcinomas (17). The present study also found that HK2 was overexpressed in CRC samples compared to normal tissue $(75.4 \%$ vs. $40 \%$, $\mathrm{P}<0.001)$, which is consistent with a malignant tumor depending on glycolysis to survive. Patra et al (18) confirmed that HK2 is required for tumor initiation and maintenance in mouse cancer models. Tumor cells are dependent on HK2 for proliferation. In the present study, we found that high HK2 expression was correlated with better PFS when treated by cetuximab plus chemotherapy compared with low expression (23.9 months vs. 6.9 monthsl; $\mathrm{P}=0.021$ ), suggesting that cetuximab plus chemotherapy will have greater efficacy in patients with high HK2 expression. HK2 expression has association with PFS but no with ORR and DCR, which may result from bias of small samples. Simultaneously, for patients treated with chemotherapy alone, HK2 had no effect on PFS. Therefore, we concluded that HK2 may be a new marker for predicting cetuximab efficacy.
In the present study, we observed an interesting phenomenon in which high LDHA expression was correlated with ORR and PFS in patients treated with chemotherapy alone compared to those treated with chemotherapy plus cetuximab. In patients treated with chemotherapy alone, high LDHA expression indicated a better ORR (72.2\% vs. $15.4 \%, \mathrm{P}=0.006)$ and PFS (18.3 months vs. 10.1 months, $\mathrm{P}=0.005)$. However, in patients treated with chemotherapy plus cetuximab, LDHA expression had no effect on ORR or PFS. We conclude that LDHA is a useful predictor for the efficacy of chemotherapy alone. Both HK2 and LDHA are key glycolysis enzymes, but they predict the efficacy of chemotherapy or cetuximab in different ways, which is an interesting phenomenon to explore. As shown in Table II, the PFS in patients receiving chemotherapy alone was $>10$ months in each subgroup, which was much better than the data shown in CRYSTAL and OPUS study $(19,20)$. In the present study, the percent of left-sided colon cancer was higher than that of right-sided colon cancer in the subgroup receiving chemotherapy alone ( $81.2 \%$ vs. $18.8 \%)$. In this subgroup, there is higher rate of male and lower $\mathrm{T}$ stage patients, with a rate of (male $71.4 \%$ vs. female $28.6 \%$ ) and T stage (T1 + T2 + T3 stage $81.2 \%$ vs. T4 stage $18.8 \%$ ). Maybe this can explain the PFS in patients receiving chemotherapy alone is $>10$ months.

In our study, we concluded that the three key glycolysis enzymes HK2, PKM2, and LDHA had no effect on patient 


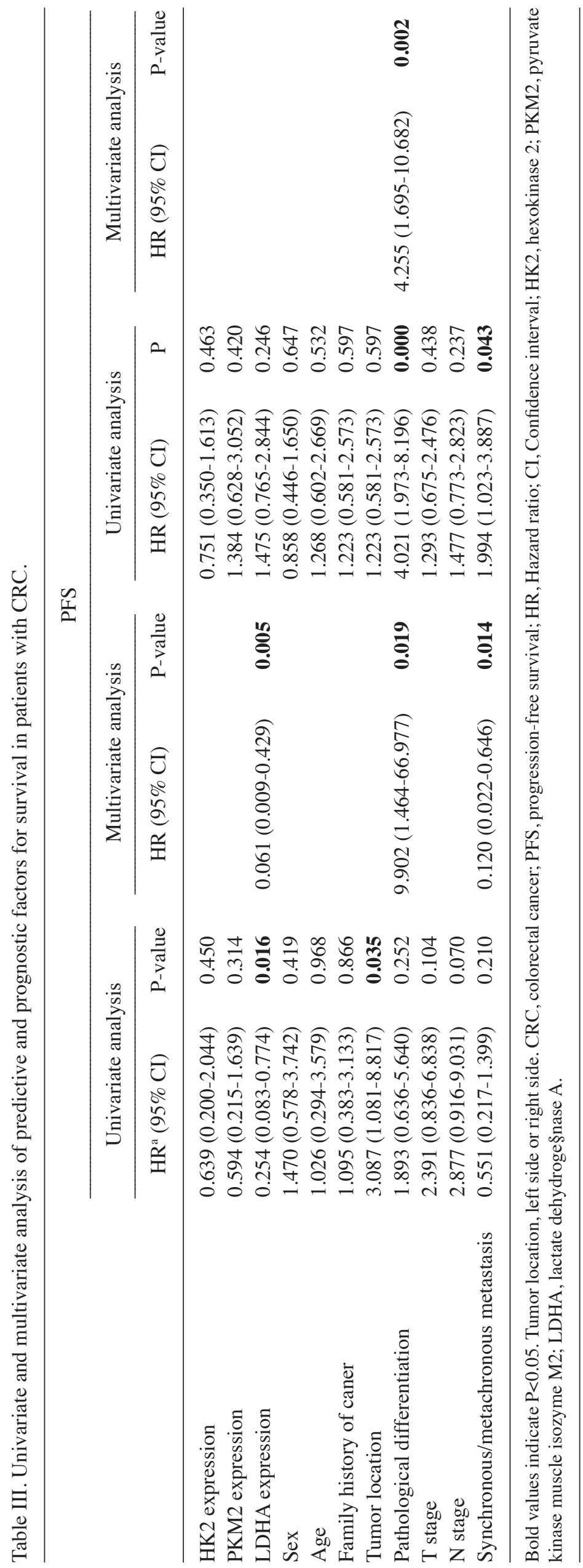


A

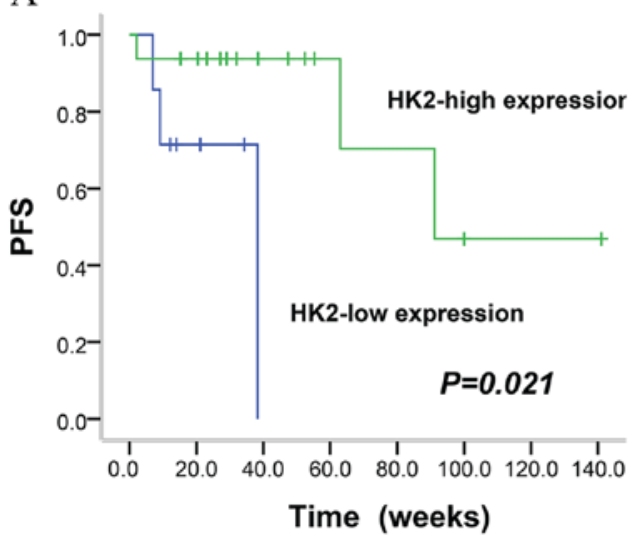

C

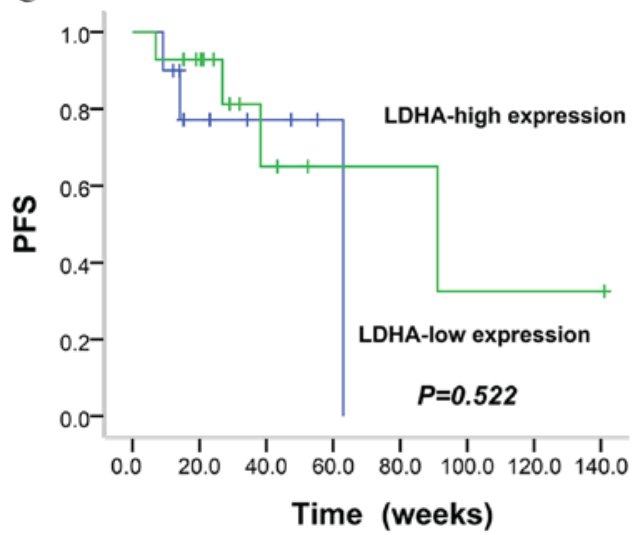

B

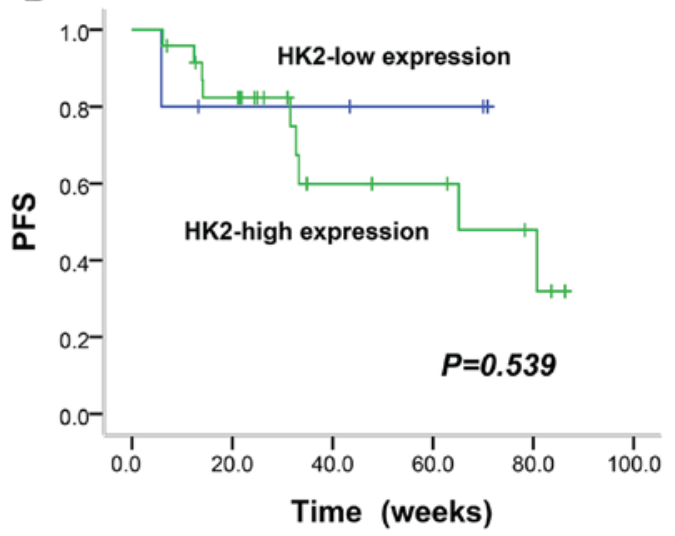

D

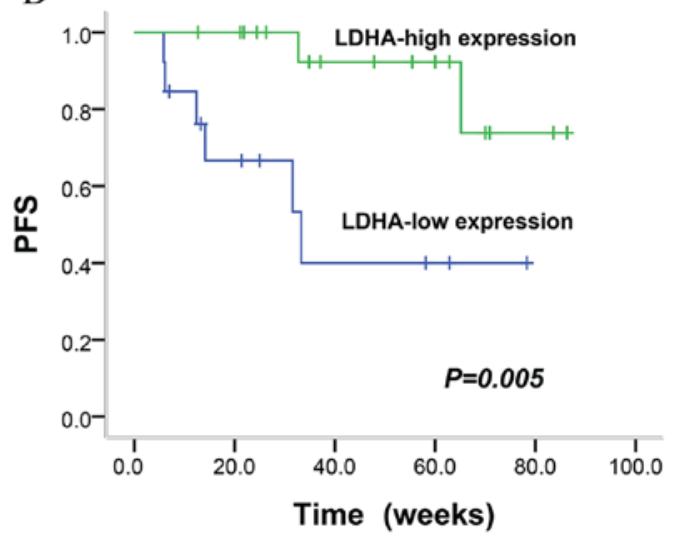

Figure 3. Kaplan-Meier curves of the PFS in CRC patients treated with chemotherapy with or without cetuximab. (A) CRC patients treated with chemotherapy plus cetuximab stratified by HK2 expression; (B) CRC patients treated with chemotherapy alone stratified by HK2 expression; (C) CRC patients treated with chemotherapy plus cetuximab stratified by LDHA expression; (D) CRC patients treated with chemotherapy alone stratified by LDHA expression. PFS, progression-free survival; CRC, colorectal cancer; HK2, hexokinase 2; LDHA, lactate dehydrogenase A.

OS. However, whether that meant that HK2, PKM2 and LDHA were not prognostic factors for mCRC treated with cetuximab remained unknown. Many studies have reported that high expression of HK2 was associated with poor survival outcomes in some tumor types, such as breast cancer (21), gastric cancer (15), non-small-cell lung cancer (22), non-Hodgkin lymphoma (23), nasopharyngeal carcinoma (13), hepatocellular carcinoma (17), cervical squamous cell carcinoma (24), and pancreatic cancer (25), among others. A meta-analysis revealed that $\mathrm{HK} 2$ overexpression was significantly associated with a worse OS and PFS in solid tumors (26). Another study reported that high $\mathrm{HK} 2$ expression was associated with reduced recurrence-free survival (RFS) in CRC patients (27). Our results regarding HK2 association with PFS or OS in CRC treated with cetuximab plus chemotherapy are not completely consistent with studies in other fields, and this discrepancy may be explained by several reasons. The first is the small number of samples in our study. Second, we studied different cancers than those in other reports. Third, we chose only mCRC cases, and our patients received similar but not identical regimens compared to those of other CRC studies. A more rigorously designed trial is needed to confirm our primary results. Additionally, our study shows that pathological differentiation is an independent prognostic factor for PFS and OS in CRC patients.

In the present study, we not only confirmed the selective upregulation of HK2 in CRC cells compared with the adjacent margin but also verified that HK2 predicts cetuximab efficacy and that LDHA predicts chemotherapy efficacy. These findings may aid physicians' decision to administer chemotherapy or cetuximab plus chemotherapy for a patient in the clinic. This is a small sample study and has some limits. The results in the present study gives us a hint: HK2 maybe a good biomarker for the treatment of cetuximab, but the final conclusion needs more study to confirm.

Our study had several shortcomings: First, it was retrospective, the patients received different therapies after first-line treatment, and the number of cases was small. Second, our study focus on HK2 expression with cetuximab efficacies, so we do not detect KRAS exon 3,4 and NRAS beyond KRAS 2. However, we identified a new predictor, HK2, for cetuximab treatment efficacy in $\mathrm{mCRC}$ and the association between HK2 and cetuximab was firstly reported up to our known, so the present study was significant in spite of small simple. On the other hand we confirmed the correlation of LDHA and chemotherapy efficacy.

\section{Acknowledgements}

The present study was supported by Guangdong Provincial Natural Science Foundation (grant no. 2017A030313685) and Guangdong Provincial Traditional Chinese Medicine Bureau of Scientific Research Projects (grant no. KY040217, 2017). 


\section{References}

1. Torre LA, Bray F, Siegel RL, Ferlay J, Lortet-Tieulent J and Jemal A: Global cancer statistics, 2012. CA Cancer J Clin 65: 87-108, 2015

2. Edwards BK, Ward E, Kohler BA, Eheman C, Zauber AG, Anderson RN, Jemal A, Schymura MJ, Lansdorp-Vogelaar I, Seeff LC, et al: Annual report to the nation on the status of cancer, 1975-2006, featuring colorectal cancer trends and impact of interventions (risk factors, screening and treatment) to reduce future rates. Cancer 116: 544-573, 2010

3. Yee YK, Tan VP, Chan P, Hung IF, Pang R and Wong BC: Epidemiology of colorectal cancer in Asia. J Gastroenterol Hepatol 24: 1810-1816, 2009.

4. Levin B, Lieberman DA, McFarland B, Smith RA, Brooks D, Andrews KS, Dash C, Giardiello FM, Glick S, Levin TR, et al: Screening and surveillance for the early detection of colorectal cancer and adenomatous polyps, 2008: A joint guideline from the American Cancer Society, the US Multi-Society Task Force on Colorectal Cancer and the American College of Radiology. CA Cancer J Clin 58: 130-160, 2008.

5. Schrag D: The price tag on progress-chemotherapy for colorectal cancer. N Engl J Med 351: 317-319, 2004.

6. Lievre A, Bachet JB, Le Corre D, Boige V, Landi B, Emile JF, Côté JF, Tomasic G, Penna C, Ducreux M, et al: KRAS mutation status is predictive of response to cetuximab therapy in colorectal cancer. Cancer Res 66: 3992-3995, 2006.

7. Heinemann V, von Weikersthal LF, Decker T, Kiani A, Vehling-Kaiser U, Al-Batran SE, Heintges T, Lerchenmüller C, Kahl C, Seipelt G, et al: FOLFIRI plus cetuximab versus FOLFIRI plus bevacizumab as first-line treatment for patients with metastatic colorectal cancer (FIRE-3): A randomised, open-label, phase 3 trial. Lancet Oncol 15: 1065-1075, 2014.

8. Alan P and Venook DN: Impact of primary $\left(1^{\circ}\right)$ tumor location on overall survival (OS) and progression-free survival (PFS) in patients (pts) with metastatic colorectal cancer (mCRC) Analysis of CALGB/SWOG 80405 (Alliance) [Abstract]. 2016 ASCO Annual Meeting 34: 3504, 2016.

9. Alan P and Venook DN: Impact of primary $\left(1^{\circ}\right)$ tumor location on Overall Survival (OS) and Progression Free Survival (PFS) in patients (pts) with metastatic colorectal cancer (mCRC): Analysis of All RAS wt patients on CALGB/SWOG 80405 (Alliance) [Abstract]. ESMO Congress 2016 [Abstract]. ESMO Congress, 2016

10. Weihua Z, Tsan R, Huang WC, Wu Q, Chiu CH, Fidler IJ and Hung MC: Survival of cancer cells is maintained by EGFR independent of its kinase activity. Cancer Cell 13: 385-393, 2008

11. Guo GF, Cai YC, Zhang B, Xu RH, Qiu HJ, Xia LP, Jiang WQ, Hu PL, Chen XX, Zhou FF and Wang F: Overexpression of SGLT1 and EGFR in colorectal cancer showing a correlation with the prognosis. Med Oncol 28 (Suppl 1): S197-S203, 2011.

12. Hanahan D and Weinberg RA: Hallmarks of cancer: The next generation. Cell 144: 646-674, 2011

13. Zhang MX, Hua YJ, Wang HY, Zhou L, Mai HQ, Guo X, Zhao C, Huang WL, Hong MH and Chen MY: Long-term prognostic implications and therapeutic target role of hexokinase II in patients with nasopharyngeal carcinoma. Oncotarget 7: 21287-21297, 2016.

14. E. Grizzle J: Continuity Correction in the $\chi 2$-Test for $2 \times 2$ Tables, 1967.
15. Sun X, Sun Z, Zhu Z, Guan H, Zhang J, Zhang Y, Xu H and Sun M: Clinicopathological significance and prognostic value of lactate dehydrogenase A expression in gastric cancer patients. PLoS One 9: e91068, 2014.

16. Girgis H,Masui O,White NM,Scorilas A,RotondoF,Seiv wright A, Gabril M, Filter ER, Girgis AH, Bjarnason GA, et al: Lactate dehydrogenase $\mathrm{A}$ is a potential prognostic marker in clear cell renal cell carcinoma. Mol Cancer 13: 101, 2014.

17. Zhang ZF, Feng XS, Chen H, Duan ZJ, Wang LX, Yang D, Liu PX, Zhang QP, Jin YL, Sun ZG and Liu H: Prognostic significance of synergistic hexokinase-2 and beta2-adrenergic receptor expression in human hepatocelluar carcinoma after curative resection. BMC Gastroenterol 16: 57, 2016.

18. Patra KC, Wang Q, Bhaskar PT, Miller L, Wang Z, Wheaton W, Chandel N, Laakso M, Muller WJ, Allen EL, et al: Hexokinase 2 is required for tumor initiation and maintenance and its systemic deletion is therapeutic in mouse models of cancer. Cancer Cell 24: 213-228, 2013.

19. Bokemeyer C, Bondarenko I, Makhson A, Hartmann JT, Aparicio J, de Braud F, Donea S, Ludwig H, Schuch G, Stroh C, et al: Fluorouracil, leucovorin, and oxaliplatin with and without cetuximab in the first-line treatment of metastatic colorectal cancer. J Clin Oncol 27: 663-671, 2009.

20. Van Cutsem E, Köhne CH, Hitre E, Zaluski J, Chang Chien CR, Makhson A, D'Haens G, Pintér T, Lim R, Bodoky G and Roh JK: Cetuximab and chemotherapy as initial treatment for metastatic colorectal cancer. N Engl J Med 360: 1408-1417, 2009.

21. Huang X, Li X, Xie X, Ye F, Chen B, Song C, Tang H and Xie X: High expressions of LDHA and AMPK as prognostic biomarkers for breast cancer. Breast 30: 39-46, 2016.

22. Koukourakis MI, Giatromanolaki A, Sivridis E, Bougioukas G, Didilis V, Gatter KC and Harris AL; Tumour and Angiogenesis Research Group: Lactate dehydrogenase-5 (LDH-5) overexpression in non-small-cell lung cancer tissues is linked to tumour hypoxia, angiogenic factor production and poor prognosis. Br J Cancer 89: 877-885, 2003.

23. Lu R, Jiang M, Chen Z, Xu X, Hu H, Zhao X, Gao X and Guo L: Lactate dehydrogenase 5 expression in Non-Hodgkin lymphoma is associated with the induced hypoxia regulated protein and poor prognosis. PLoS One 8: e74853, 2013.

24. Huang X, Liu M, Sun H, Wang F, Xie X, Chen X, Su J, He Y, Dai Y, Wu H and Shen L: HK2 is a radiation resistant and independent negative prognostic factor for patients with locally advanced cervical squamous cell carcinoma. Int J Clin Exp Pathol 8: 4054-4063, 2015.

25. Ogawa H, Nagano H, Konno M, Eguchi H, Koseki J, Kawamoto K, Nishida N, Colvin H, Tomokuni A, Tomimaru Y, et al: The combination of the expression of hexokinase 2 and pyruvate kinase M2 is a prognostic marker in patients with pancreatic cancer. Mol Clin Oncol 3: 563-571, 2015.

26. Liu Y, Wu K, Shi L, Xiang F, Tao K and Wang G: Prognostic significance of the metabolic marker hexokinase-2 in various solid tumors: A meta-analysis. PLoS One 11: e0166230, 2016.

27. Hamabe A, Yamamoto H, Konno M, Uemura M, Nishimura J, Hata T, Takemasa I, Mizushima T, Nishida N, Kawamoto K, et al: Combined evaluation of hexokinase 2 and phosphorylated pyruvate dehydrogenase-E1 $\alpha$ in invasive front lesions of colorectal tumors predicts cancer metabolism and patient prognosis. Cancer Sci 105: 1100-1108, 2014

This work is licensed under a Creative Commons Attribution-NonCommercial-NoDerivatives 4.0 International (CC BY-NC-ND 4.0) License. 